Title

\title{
SAFELY ENHANCING SURGICAL WARD PATIENT FLOW IN THE BED SHORTAGE ERA BY A MODIFIED "SAFER SURGERY RED2GREEN" MULTI-DISCIPLINARY BOARD ROUND. A QUALITY IMPROVEMENT RESEARCH REPORT.
}

Roberto Valente ${ }^{1,2,3}$, Lorna Stanton ${ }^{1}$, Gregorio Santori ${ }^{4}$, Ajit Abraham ${ }^{1}$, Mohamed A Thaha ${ }^{1,5}$.

(1) Department of General Surgery, Royal London Hospital, Barts Health NHS Trust, Whitechapel, London E1 1BB, UK

(2) Division of Surgery and Interventional Science, University College London, London, UK

(3) Department of Surgery, Policlinico S. Martino, Genoa, Italy

(4) Department of Surgical Sciences and Integrated Diagnostics, University of Genoa, Genoa, Italy

(5) Blizard Institute, National Bowel Research Centre, Barts and the London School of Medicine and Dentistry, Queen Mary University of London, London, UK

Roberto Valente and Mohamed A Thaha are joint senior authors

\section{Joint Corresponding Authors}

Roberto Valente MD, PhD, MSC, FEBS(HPB), FRCS(Eng)

Division of Surgery and Interventional Science, University College London, London, UK

Email:r.valente@ucl.ac.uk,roberto.valente@nhs.net

Mohamed A Thaha MBBS, PhD, FRCS(Glasg), PG Cert HIt Econ

Royal London Hospital, Barts Health NHS Trust

Blizard Institute, Barts and the London School of Medicine \& Dentistry

Queen Mary, University of London, London, UK

Email: $\underline{\text { m.a.thaha@qmul.ac.uk }}$

\section{Abstract}

\section{Background}

Acute hospital bed shortage is a serious concern worldwide, constantly involving high-dependency units (HDU), where the non-availability of postoperative beds results in surgery cancellation. In the acute medicine context, the SAFER Red2Green initiative has shown to enhance patient flow. 


\section{Local problem}

At the Royal London hospital, in 2016, hospital-initiated cancellations peaked at over $50 \%$ weekly due to the inability of high dependency Units (HDU) to discharge step-down patients to the general surgical wards, where bed occupancy was close to $100 \%$ and the average length of stay was stable on average close to 7 (+/-8.6) days.

\section{Methods}

This was a service improvement research to enhance patient flow which adapted the SAFER Red2Green model to a surgical ward (SAFER Surgery Red2Green). This before-after study involving all 2017 digestive surgery admissions was divided into a three-month feasibility phase followed by a nine-month pilot phase, versus the year 2016 (pre-intervention). Outcome measures: weekly discharges, length of stay (LOS), surgery cancellations, feasibility of a "theatre go" policy, HDU stepdowns, 30-day readmissions.

\section{Interventions}

1) Systematic communication of key care plan from the afternoon ward rounds by surgical teams to the nurse in charge; 2) 10 AM Monday-to-Friday multi-disciplinary senior-team daily board round, addressing updated key care plan aimed at early discharges, appropriateness of each inpatient day, causes of delays; 3 ) hospital and site managers weekly attendance.

\section{Results}

At three months: $+67 \%$ discharges/week ( $p=0.001),-20 \%$ LOS $(p=0.023),+21 \%$ HDU step-downs, $(p=0.205)$. At one year: $+10.7 \%$ HDU step-downs ( $p=0.197)$, increased probability of earlier discharge $(p=0.023),-60 \%$ hospital-initiated cancellations from 38 to 15 ( $p>=1)$, a "Theatre go" policy has been active since month 6 . Failed discharges kept at $1.3 \%$. The MDT board round staff satisfaction rate was over $80 \%$, with key actors' attendance over $75 \%$.

\section{Conclusions}

The SAFER Surgery R2G model safely enhanced patient flow and reduced cancellations and unnecessary nurse staff time. It requires senior medical and nursing commitment, however, is designed for any surgical specialty, and has proven sustainable. It warrants further validation.

\section{Background}

\section{Local problem}

Hospital bed shortage has long been a concern for the NHS and at times has contributed to serious consequence for patients. In January 2017, following the death of two patients whilst waiting in a trolley for an in-patient bed in a NHS hospital in England, the newspaper, Guardian, published a controversial article highlighting the bed crisis in the English NHS. The report criticised that the NHS is 'broken' and is in a 'humanitarian crisis' $[1,2]$. Our hospital is a major trauma centre for London where the 'Helicopter Emergency Medical Service (HEMS) is based. The increasing demand for emergency beds result in 'capacity saturation' and this along with a slower patient flow causes an 
upstream pressure delaying 'high dependency unit' (HDU) step-downs. The consequent nonavailability of postoperative surgical beds frequently results in 'on the day' cancellation of planned surgery for benign and cancer cases. Such a complex situation in bed shortage has now become overwhelming due to the COVID 19 pandemic, as increased ICU staff needs during SARS peaks have disrupted surgical planning $[3,4]$ postponing routine operations, creating impressive backlog consequences [4] and making patients' prioritisation [5,6] and enhanced recovery [7] advocated for elective surgery.

\section{Available knowledge and rationale}

Structured ward rounds enhance quality of care [8] [9], and the assessment of key information with involvement of senior medical and nursing staff in the patients care plan increases critical patient safety [10]. Use of a ward round template improves compliance to crucial safety parameters due to their discussion during ward rounds [8][11].

In the late nineties, multidisciplinary (MDT) bedside rounds in surgery have been reported (5) to enhance team working with improvement in length of stay (LOS) and costs. Unfortunately, these models often require rearrangement of interdisciplinary job plans and different role interactions [13]. Senior assessment during daily ward round has proven to be clinically beneficial and is cost effective $[14,15]$. Board rounds instead of ward rounds can facilitate such daily senior MDT assessment [16].

In the context of centralised and super-specialised patient care, patient flow has recently become a critical issue in hospital management [17], and delayed discharges have shown to worsen hospital bed occupancy and patient quality of care [18].

The SAFER patient flow bundle [19] and Red2Green days (SAFER R2G) model have been introduced, addressing the enhancement of patient flow by introducing systematic daily senior review, multidisciplinary team (MDT) work towards an expected discharge date with clear clinical criteria for discharge, optimisation of inpatient flow, and review of causes for delayed discharges [20]. Since its introduction [21], the approach has found an expanding application in the NHS [22]. To our knowledge, the SAFER R2G model has never been applied in a general surgical environment, but it is expected to work given the rationale described above.

\section{Aim}

The aim of this service improvement research was to design, introduce and test a novel MDT surgical board round methodology, based on the SAFER R2G approach (SAFER Surgery R2G), to safely improve a quality surgical ward round and increase the patient flow.

\section{Methods}

\section{Context}

The 25-bed general surgery in-patient ward at our hospital, which provides inpatient care for digestive surgery patients belonging to upper gastrointestinal, colorectal and hepato pancreatobiliary specialities. The service has 11 consultants and 18 junior doctors, and the ward has its own resident nursing team and a ward manager. The general surgery department runs a 24-hour consultant emergency on-call rota from mid-day to mid-day (e.g. Consultant A - starts on-call at 12:00 on Monday and finishes on-call on 12:00 on Tuesday. They undertake a post-take ward round at $8 \mathrm{AM}$ on Tuesday and the board round were made part of the post-take activity and starting at a fixed time (10:00 AM)). On weekdays, every morning and afternoon each consultant firm performs a 
separate ward round led by the specialist registrar $(\mathrm{SpR})$ for their own patients. Physiotherapists, social workers, pharmacists, and other multi-specialty staff attend the ward daily and provide their input separately. Patients for planned surgery are admitted to a separate ward but are stepped down from the acute critical care unit (ACCU) to the ward.

The division of surgery ran several initiatives to improve patient flow before the start of SAFER Surgery R2G board round on $1^{\text {st }}$ January 2017 . These included

1. A "complex discharge" facilitation project by a dedicated team liaising with referring GPs and aftercare units.

2. A "theatre go" policy, which aimed to start the first operation in every list even when the postoperative bed has not been identified.

3. A pre-discharge step-down policy towards a lower intensity area and an increased utilisation of discharge lounge

\section{Intervention}

We introduced and assessed our board round methodology following the methods described below, based on a two-step feasibility-pilot approach, involving all the key actors of the patient clinical care on the ward.

\section{Study design}

This is a before-after study, comparing year 2016 (pre-intervention) versus year 2017 (postintervention). The service improvement project was approved by the division of surgery and perioperative care, and is reported according to the SQUIRE 2.0 standards (16). The study is divided into three phases:

1. Clinical protocol definition

2. Feasibility study and protocol refinement

3. Pilot study

\section{Clinical protocol definition}

The clinical protocol for the study was designed in November 2016 based on available evidence and experiences, Barts Health Trust policies and projects, and by adapting the SAFER R2G to the surgical environment. The general surgery departmental audit meeting attended by all grades of medical staff approved the preliminary protocol in December 2016, which had the following key principles:

- systematic communication of key care plan from the afternoon ward rounds by each surgical firm to the nurse in charge so that this information can be included in the evening handover to the night team;

- daily (Monday to Friday) multi-disciplinary senior team board round at 10:00 AM addressing:

- updated key care plan aimed at early discharges;

- MDT appropriateness evaluation of each day;

- twice weekly attendance of the board round by the hospital management and site managers;

- weekly review of delays;

- the four named authors (three consultant surgeons AA, RV, MAT and a specialist nurse LS) led the project. 


\section{Feasibility study}

The three months' feasibility phase of the structured board rounds commenced in January 2017 and was chaired by the three consultant surgeons and specialist nurse leading the project. Monthly departmental audit meetings were used to provide progress reports to the entire department.

All admissions to the general surgery ward from January $1^{\text {st }} 2017$ were included in this feasibility study, irrespective of the base specialty. This included some of the "outlier patients" who were under the care of vascular and trauma surgery, and orthopaedics, hosted in emergency in the general surgery ward due to bed shortage in the respective services. However, only general surgery consultants participated in the board rounds.

The study team assessed the safety and feasibility of the project at weekly team meetings and made early revisions of the outcome measures. The final study protocol was presented to and approved at a consensus meeting at the departmental audit day (Table 1).

\section{Pilot study}

The 9-month pilot phase of the study was conducted from $1^{\text {st }}$ April 2017 to $31^{\text {st }}$ December 2017 . The on-call consultant from the night before chaired the daily MDT board round so that this activity followed the post-emergency take ward rounds. The prospective audit of the MDT board round collated information on performance progress and attendance by different team members and of the communication of the daily surgical key care plan to the nurse in charge. The 12-months afterintervention period ended on 31 December 2017.

\section{Study of the interventions}

A set of primary and secondary measures were chosen to assess the impact of the SAFER Surgery R2G model, while addressing its sustainability. These were monitored weekly, to ensure their accuracy and completeness. We compared the 12 months before versus 12-months after intervention periods, pairing weekly values of the two groups to reduce the impact of seasonal changes in the demand.

\section{Measures}

\section{Primary outcome measures (of the intervention)}

1) Overall and weekly ward discharges and probability of discharge, chosen as overall flow measure; this was automatically computed by the hospital admission systems with no gaps.

2) Length of stay of general surgery patients (from admission to discharge), as an overall measure of the patient pathway.

\section{Secondary outcome measures (to determine the impact of contributing contextual elements)}

1) Elective surgery cancellations due to ACCU (ICU/HDU) and ward bed non-availability (monthly totals).

2) Feasibility of a "theatre go" policy (yes/no) as a marker of a stabilised trend of sufficient postoperative care capacity. Prior to analysis we have confirmed data completeness and accuracy.

3) ACCU (ICU/HDU) step-downs to general surgery ward. 
Quality control measures: 30 -day readmission rates (safety), ward bed capacity utilisation (efficiency).

Qualitative assessment. Satisfaction rates by MDT board round participants per category. A qualitative measure on the project effectiveness and work environment appreciation, monthly providing a 1-5 score (mean \%) to the following question: "Are you satisfied by the MDT board round as an occasion to share and action upon the patients' issues, and expedite their progress safely?".

\section{Statistical analysis}

The results are expressed as mean \pm standard deviation, median, counts or percentages. The Shapiro-Wilk test was used to assess normal distribution of continuous variables. Categorical variables were analysed with $\chi 2$ test or Fisher's exact text when appropriate. Comparisons between continuous variables were performed with the Mann-Whitney-Wilcoxon rank sum test. Cumulative probability of discharge was evaluated by using Kaplan-Meier product-limit estimator, with log-rank test to compare time-event curves. Statistical significance was assumed in each two-tailed test with $p$ value $<0.05$. Statistical analysis was carried out by using the $R$ software/environment (version 3.6.3; R Foundation for Statistical Computing. Vienna, Austria).

\section{Ethical considerations}

The project was commissioned by the hospital trust and approved by the Divisional and Service leading groups and discussed in the standard governance meetings throughout. No formal ethical approval was deemed necessary, given the study was designed as a service improvement exercise with no change in the direct clinical care. None of the authors have any competing or conflicting interest in relation to the present study.

\section{Results}

\section{Months 1-3: feasibility phase}

At the end of the feasibility phase (month 3) 275 new patients (114 female / 161 male) of 57 (+/18.3) years of age. No major adverse events were recorded as related to the intervention. Figure 1 shows the evolution over time of ward discharges in weekly counts. Compared to the same period of the previous year the overall patient flow had significantly increased with a mean discharges' count difference of 8.5 weekly discharges $(+67 \%, p=0.001)$, median LOS reduced from 5 to 4 days $(-20 \%$, $p<0.001)$, and a significantly increased probability of discharge (log rank $p=0.002)$. Intensive Care Unit / High Dependency Unit (ICU/HDU) step-downs increased from 81 to $95(+18.5 \%, p=0.043)$ each week.

Following the feasibility phase of the study, we initially recorded a $60 \%$ attendance rate for consultants' chairing the SAFER Surgery R2G MDT board rounds, with an average $75 \%$ of attendance by all actors. The monthly progress review meeting identified that such a low rate was mostly reported due to conflicting commitments for the consultants, precluding them from attending the MDT board rounds. The issue was addressed by incorporating the SAFER Surgery R2G MDT board rounds within the post emergency-on-call take rota. Over $75 \%$ satisfaction rate (answer "Yes" to the qualitative question) was recorded by all staff speciality representatives attending the MDT board rounds.

A larger number of patients from specialities other than general surgery such as vascular surgery, trauma, and neurosurgery, and to lesser extent patients from a medical speciality were admitted to 
the ward as 'outliers'. Such non-general surgery admission steadily accounted for over $15 \%$ of the overall ward occupation and was deemed an unavoidable consequence of the bed overnight availability for emergency admissions.

\section{Months 4-12: Pilot phase}

Along with the pilot phase progress, we have observed some changes that might potentially be interacting with the project setting. These include the expansion of the consultant pool chairing the board round MDT meeting, the contemporary wider hospital flow-enhancing initiative, and the extension of the SAFER R2G piloted model to other surgical wards, sharing the quality improvement team resources.

The pilot progressed consistently until the end of the monitored period in December 2017. Senior consultant attendance occurred in approximately $75 \%$ of the cases, and inconsistently structured communication between surgical teams and Nurse in Charge were observed in about one third of the afternoons, despite specific reminders and acknowledgment at the Service Audit meetings.

Results for the full 12 -months period are shown in Table 2.Table 2 The improvements observed during the feasibility phase reduced their immediate magnitude, however stabilised on a significantly positive trend throughout the year of primary outcome measures: overall discharges increased from 954 to $2032(+8.1 \%)$ weekly mean ward discharges increased from 14 to $16(+14 \%$, $p=0.027)$, and probability of discharge increased on both overall and surgical ward length of stay (log rank $p=002, p=0.014$ ), while mean ward length of stay (LOS) decreased from 6.9 (8.6) to 6.1 (7.4) (i.e. $-13 \%)$ close to - however not reaching - significance $(p=0.062)$, even though emergency/elective admission rate significantly increased from $1.01 / 1.27+25 \%(p=0.002)$ with the increased overnight bed availability for emergency admission.

Secondary outcome measures. Total elective surgery cancellations due to ICU/HDU and ward bed non-availability dropped from 38 to $15(-60.5 \%, p>=1)$, allowing major elective cases to proceed, and in June 2017 a "Theatre-go policy" was established, as elective cases were allowed to proceed even when an ACCU bed was not available at the start of surgery. The faster ACCU beds admission and step-down flow measured a $+9.3 \%$ of total cases, from 345 to $375(p=0.197)$, with a decreased average step-down weekly average delay from $0.7(1.1)$ to $0.6(0.9)$ days $(p=0.761)$.

A 30-day re-admission increase of $0.4 \%$ from $9(0.9 \%)$ to $14(1.3 \%)$ cases $(p=0.390)$. High satisfaction $(>75 \%)$ rates were recorded by all MDT staff categories, reported as mainly referred to enhanced teamwork and faster decisions on clinical plans. Medical attendance was the hardest to achieve (consultant 75\%, Registrar 60\%), however average attendance was about $80 \%$.

\section{Discussion}

\section{Summary}

To our knowledge this is the first report of an original modification of the SAFER R2G model, applied to a NHS surgical service. When used in our study, the model improved discharge rates and probability of earlier discharge $(p<0.05)$ without compromising patient safety, and contributing to solve major capacity issues in the context of saturated bed occupancy, such as reducing cancellations and allowing a "theatre-go" policy. The acute bed crisis has consistently represented a 
major issue in the recent years, and further dramatically has during the COVID-19 pandemic, as nursing staff relocation makes beds available even less.

\section{Interpretation}

The application of our SAFER Surgery R2G to model has shown evident results, which are reasonably confirmed by the magnitude and the time-correspondence of the changes in our surgical ward observed during the early phase, and the maintained statistical significance of our primary endpoint results throughout the full study period. In the absence of other evidence from other researchers, our results might represent a basis for comparisons and modifications of our model. The satisfactory (non-quantitative) feedback from our staff indicates that the SAFER Surgery R2G model has positively impacted on our everyday work, contributing to safely increase patient flow, as well as teamwork and clinical leadership on the surgical ward. Additionally, ward staff reported that the easier access to care plan information provided by the structured handover and board round free up staff time further, especially nursing time. The structure and clarity of information relayed also allowed for more efficient actions being taken promptly.

The improvements we have measured were particularly evident during the initial feasibility phase, rather than during the subsequent 9-months pilot phase. We attribute this difference primarily to the unavoidable near-to-100\% saturation of bed capacity by 'outlier' patients (on the ward course of whom our MDT board round had no effect), and to a poor compliance of the community-based environment to repatriations, community care, rehabilitation, etc., preventing from improving the flow further by making discharge of clinically fit patients more difficult. Lastly, we experienced the difficulty to reach $100 \%$ attendance by the senior clinicians, hence maintaining some degree of delay in taking prompt non-urgent decisions.

The enhancement in patient ward flow obtained by our SAFER Surgery R2G model has allowed an "upstream" benefit in other hospital areas, significantly increasing bed availability for emergency cases otherwise stationing in the Accident and Emergency areas. From the ICU/HDU domain, allowing faster flow makes more efficient use of such expensive resources by reducing the delay of step-downs of clinically fit patients, and further involves theatres. In our experience, the latter area has benefitted from the $60 \%$ drop of hospital-initiated cancellations, causing a relevant cost for both patients and services.

\section{Limitations}

The internal validity of our study is affected by the change over time in the complex hospital organisation our project was run. To reduce their impact, we have completed a 12 months' study and observed the results over time, pairing each year-week to minimise seasonal changes.

More research is, however, needed to confirm the external validity of our pilot work, due to the several confounding and interactive factors we have encountered, likely unavoidable in such a large organisation. However, we have adjusted our analysis on "outliers" patients, and have chosen generic and non-specialty dependant criteria to maximise reproducibility in other hospitals. We have not measured more precise parameters such as time from clinical fitness to actual discharge, morning discharges, totals of red2green days etc., as these outcome measures proved not available at the end of the feasibility study. The wider use of electronic data systems may be the step forward to refine such measurements [24]. 
The need of leadership commitment represents another limitation of the application of the SAFER Surgery R2G model. A consistent, senior level clinical, nursing and management commitment was required to sustain and maximise the results observed in this study. Although it may be hard to keep the teams motivated in the current context of resource saturation, this challenge must be kept on in order to maximise the potential of our SAFER Surgery R2G model in terms of sustained benefit.

\section{Conclusions}

The adoption of the SAFER Surgery R2G model, in principle designed for any inpatient surgical specialty. It has allowed significant improvements in patient flow in our general surgery department, allowing faster hospital stays, reduced cancellations, more efficient ICU/HDU beds, and more bed availability for emergency step-downs from A\&E. Staff also described enhanced patient safety and quality of care, capacity management, and teamwork, due to earlier and multidisciplinary senior decision making. The model has proven to be sustainable, and in principle able to deliver relevant benefits across more services and hospitals. However, the SAFER Surgery R2G model requires committed leadership by the senior staff. Further applications are required to validate our pilot results, such as a multicentre service improvement research.

\section{Funding}

The project was run within NHS staff working hours, with no additional external fund.

\section{References}

1 Campbell D, Morris S, Marsh S. NHS faces "humanitarian crisis" as demand rises, British Red Cross warns. The Guardian. 2017.http://www.theguardian.com/society/2017/jan/06/nhs-faceshumanitarian-crisis-rising-demand-british-red-cross (accessed 24 May 2018).

2 O'Dowd A. Doctors condemn need for Red Cross to step in to aid NHS in "humanitarian crisis." $B M J$ 2017;356:j127. doi:10.1136/bmj.j127

3 Nepogodiev D, Bhangu A. Elective surgery cancellations due to the COVID-19 pandemic: global predictive modelling to inform surgical recovery plans. Br J Surg Published Online First: 12 May 2020. doi:10.1002/bjs.11746

4 Maringe $C$, Spicer J, Morris M, et al. The impact of the COVID-19 pandemic on cancer deaths due to delays in diagnosis in England, UK: a national, population-based, modelling study. Lancet Oncol 2020;21:1023-34. doi:10.1016/S1470-2045(20)30388-0

5 Déry J, Ruiz A, Routhier F, et al. Patient prioritization tools and their effectiveness in nonemergency healthcare services: a systematic review protocol. Syst Rev 2019;8:78. doi:10.1186/s13643-019-0992-x

6 Valente R, Di Domenico S, Mascherini M, et al. A new model to prioritize waiting lists for elective surgery under the COVID-19 pandemic pressure. Br J Surg 2021;108:e12-4. doi:10.1093/bjs/znaa028 
7 Wainwright TW. Enhanced Recovery after Surgery (ERAS) for Hip and Knee Replacement-Why and How It Should Be Implemented Following the COVID-19 Pandemic. Medicina (Mex) 2021;57. doi:10.3390/medicina57010081

8 Pucher PH, Aggarwal R, Qurashi M, et al. Randomized clinical trial of the impact of surgical wardcare checklists on postoperative care in a simulated environment. BJS;101:1666-73. doi:10.1002/bjs.9654

9 Pucher PH, Aggarwal R, Darzi A. Surgical Ward Round Quality and Impact on Variable Patient Outcomes. Ann Surg 2014;259:222. doi:10.1097/SLA.0000000000000376

10 Massey D, Aitken LM, Chaboyer W. What factors influence suboptimal ward care in the acutely ill ward patient? Intensive Crit Care Nurs 2009;25:169-80. doi:10.1016/j.iccn.2009.03.005

11 Tranter-Entwistle I, Best K, lanev R, et al. Introduction and validation of a surgical ward round checklist to improve surgical ward round performance in a tertiary vascular service. ANZ J Surg 2020;90:1358-63. doi:10.1111/ans.15899

12 Felten S, Cady N, Metzler MH, et al. Implementation of collaborative practice through interdisciplinary rounds on a general surgery service. Nurs Case Manag Manag Process Patient Care 1997;2:122-6.

13 May;13:311-317 JHM 2018. Improving Teamwork and Patient Outcomes with Daily Structured Interdisciplinary Bedside Rounds: A Multimethod Evaluation. J Hosp Med 2018;13. doi:10.12788/jhm.2850

14 Geary S, Cale D-D, Quinn B, et al. Daily Rapid Rounds: Decreasing Length of Stay and Improving Professional Practice. JONA J Nurs Adm 2009;39:293-8. doi:10.1097/NNA.0b013e3181a72ab8

15 Ahmad A, Weston PJ, Ahmad M, et al. A cost-benefit analysis of twice-daily consultant ward rounds and clinical input on investigation and pharmacy costs in a major teaching hospital in the UK. BMJ Open 2015;5:e007367. doi:10.1136/bmjopen-2014-007367

16 Shabbir A, Wali G, Steuer A. Four Simple Ward Based Initiatives to Reduce Unnecessary InHospital Patient Stay: A Quality Improvement Project. BMJ Open Qual 2015;4:u208974.w3661. doi:10.1136/bmjquality.u208974.w3661

17 Catalyst N. What Is Patient Flow? NEJM Catal Published Online First: 1 January 2018.https://catalyst.nejm.org/doi/abs/10.1056/CAT.18.0289 (accessed 4 Oct 2020).

18 Majeed MU, Williams DT, Pollock R, et al. Delay in discharge and its impact on unnecessary hospital bed occupancy. BMC Health Serv Res 2012;12:410. doi:10.1186/1472-6963-12-410

19 SAFER patient flow bundle: ward rounds | NHS Improvement. https://improvement.nhs.uk/resources/safer-patient-flow-bundle-ward-rounds/ (accessed 5 Oct 2020).

20 The SAFER patient flow bundle and Red2Green days approach | NHS Improvement. https://improvement.nhs.uk/resources/safer-patient-flow-bundle-and-red2green-daysapproach/ (accessed 21 Jun 2018). 
21 Safer, faster, better: transforming urgent and emergency | NHS Improvement. https://improvement.nhs.uk/resources/safer-faster-better-transforming-urgent-andemergency/ (accessed 21 Jun 2018).

22 safer red2green | Evidence search | NICE.

https://www.evidence.nhs.uk/search?q=safer+red2green\&Route=search\&ps=100 (accessed 5 Oct 2020).

23 Ogrinc G, Davies L, Goodman D, et al. SQUIRE 2.0 (Standards for QUality Improvement Reporting Excellence): Revised Publication Guidelines from a Detailed Consensus Process. Can J Diabetes 2015;39:434-9. doi:10.1016/j.jcjd.2015.08.001

24 Alamri Y, Frizelle F, Al-Mahrouqi $\mathrm{H}$, et al. Surgical ward round checklist: does it improve medical documentation? A clinical review of Christchurch general surgical notes. Anz J Surg 2016;86:878-82. doi:10.1111/ans.13425 


\section{Tables}

Table 1 Board round clinical protocol

\begin{tabular}{|c|c|}
\hline Ward round & $\begin{array}{ll}\text { - } & \text { Twice daily registrar led: 8:00, 16:00 } \\
\text { - } & \text { Twice weekly consultant led }\end{array}$ \\
\hline \multirow[t]{3}{*}{$\begin{array}{l}\text { Daily communication } \\
\text { Surgical team }=>\text { Nurse in } \\
\text { charge }\end{array}$} & $\begin{array}{l}\text { PM Junior doctors with registrar supervision, handover to Nurse in } \\
\text { Charge by } 3 \text { PM the following handover items }\end{array}$ \\
\hline & $\begin{array}{l}\text { 1. Named consultant } \\
\text { 2. Current reason for admission ( } 15-20 \text { words max) } \\
\text { 3. Scheduled actions for tomorrow } \\
\text { 4. Revised discharge date }\end{array}$ \\
\hline & - \\
\hline \multirow[t]{5}{*}{ Mon-Fri board round } & Daily 10:00 - 10:30 \\
\hline & Led by Nurse in charge \\
\hline & Chaired by post-take consultant \\
\hline & Attended by physiotherapist and social worker, community liaison \\
\hline & $\begin{array}{l}\text { Once weekly attended by service manager, matron nurse, } \\
\text { divisional patient flow nurse coordinator }\end{array}$ \\
\hline \multirow[t]{6}{*}{ For each patient discussing } & - $\quad$ The clinical handover 1-4 items \\
\hline & - $\quad$ Discharge plan within 24 hours \\
\hline & - $\quad$ Physiotherapy need \\
\hline & - $\quad$ Likelihood of complex discharge team involvement \\
\hline & - $\quad$ Need for aftercare package \\
\hline & - $\quad$ Red or Green day \\
\hline \multirow[t]{4}{*}{ Green day definition } & All actions scheduled for the day are done or \\
\hline & At least one intervention done on the day \\
\hline & \\
\hline & \\
\hline
\end{tabular}




\begin{tabular}{|c|c|c|c|}
\hline & $2016(N=954)$ & $2017(\mathrm{~N}=1032)$ & Difference \\
\hline Age (years) & $56.6(18.4)$ & $60(18.2)$ & $p=0.108$ \\
\hline Gender (Female/Male) & $401 / 522$ & $491 / 532$ & $p=0.045$ \\
\hline \multicolumn{4}{|l|}{ Specialty service } \\
\hline Outliers & 163 & 179 & \multirow{2}{*}{$p=0.486$} \\
\hline General Surgery & 760 & 844 & \\
\hline General & 276 & 293 & \multirow{4}{*}{$P=0.290$} \\
\hline UGI & 13 & 7 & \\
\hline$H P B$ & 206 & 225 & \\
\hline Colorectal & 265 & 319 & \\
\hline Elective / Emergency & $475 / 479$ & 455 / 577 & $p=0.002$ \\
\hline \multicolumn{4}{|l|}{ Service patient flow } \\
\hline $\begin{array}{r}\text { Ward discharges: weekly } \\
\text { mean (SD) }\end{array}$ & $14(+/-4.1)$ & $16(+/-4.2)$ & $p=0.027$ \\
\hline $\begin{array}{r}\text { Ward length of stay (days): } \\
\text { mean (SD) }\end{array}$ & $6.9(8.6)$ & $6.1(7.4)$ & $p=0.062$ \\
\hline ICU/HDU Step-downs & 342 & 375 & $p=0.197$ \\
\hline $\begin{array}{r}\text { Average delay (days): } \\
\text { mean (SD) }\end{array}$ & $0.7(1.1)$ & $0.6(0.9)$ & $P=0.761$ \\
\hline Cancellations & 38 & 15 & $p>=1$ \\
\hline Theatre Go Policy & No & Yes & - \\
\hline Failed discharges (total) & $9(0.9 \%)$ & $14(1.3 \%)$ & $p=0.390$ \\
\hline
\end{tabular}


Figures

Figure 1. Weekly discharge rates

Weekly discharge rates

30

25

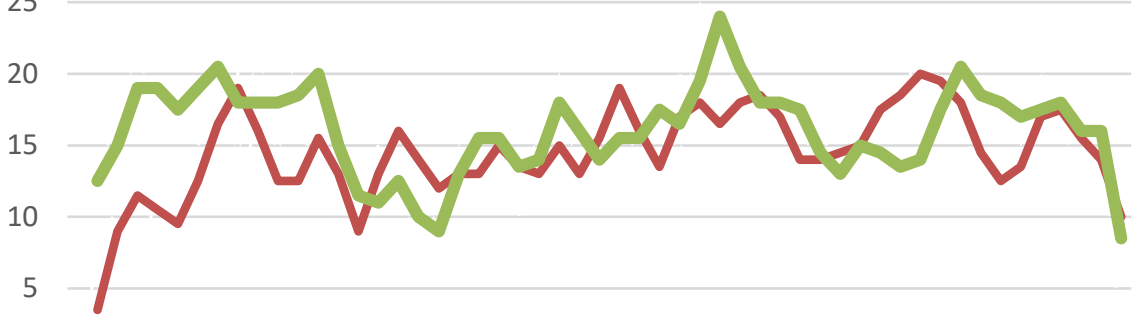

0

135779611131517192123252729313335373941434547495153

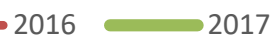


Figure 2. Probability of discharge

\section{A: full admission length of stay \\ B: surgical ward length of stay}

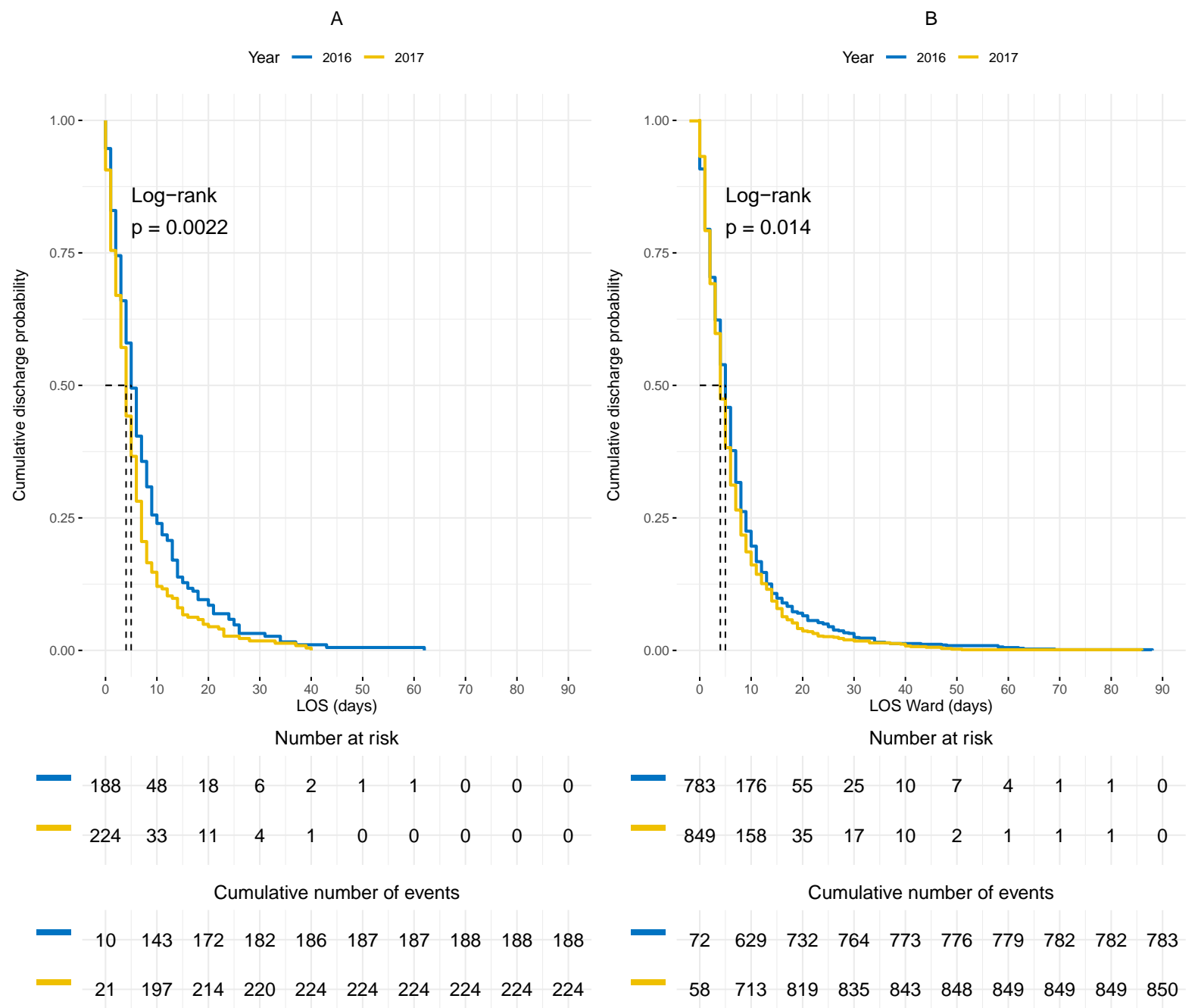

